\title{
An Efficient Texture Classification Technique Based on Semi Uniform LBP
}

\author{
Dr. Y venkateswarlu ${ }^{1}$, Dr. U Ravi Babu ${ }^{2}$, Ch Aneel Kumar ${ }^{3}$ \\ ${ }^{I}$ Professor and HOD, Department of CSE, GIET Engg. College, RJY, AP, India \\ ${ }^{2}$ Professo, Department of CSE, MREC, Secendrabad, TS, India \\ ${ }^{3}$ Software Engineer, SMACVY Innovative Solutions Pvt Ltd, Rajahmundry-AP, India
}

\begin{abstract}
Local Binary Pattern' (LBP) operator has been proved to be a theoretically simple yet very effective statistical texture descriptor in many areas. One of the main disadvantage of $L B P$ is they are more sensitive to even a simple noise, this causes a change in LBP pattern. A LBP can generate 256 (0 to 255) different patterns. These patterns are grouped into 59 uniform and 197 nonuniform LBP patterns. Most of the researchers have considered only uniform patterns for texture classification due to their small numbers and claiming that most of the textures are dominated by only uniform LBP's. But after a careful, critical and significant study on the literature, ULBP have some shortcomings: they discard some important texture information, suffer much from non-monotonic illumination variation and do not describe the stochastic characteristics of texture efficiently and sensitive to noise. To overcome this, the present paper defined 'Semi Uniform LBP' (SULBP). The proposed method has a significant role in efficient texture classification and analysis.
\end{abstract}

Keywords: Local Binary Pattern, Semi Uniform LBP, texture analysis and classification

\section{Introduction}

Local Binary Pattern (LBP), first proposed in Ojala, Pietikainen, and Maeenpaa (2002) [1]. it is one of the most widely used descriptors because of it works on different lighting conditions and also computational complexity is low. It is less sensitive to changes in illumination than many descriptors. LBP has been extensively studied by Ahonen, etal 2006 [19], Heikkilä \& Matti Pietikäinen, 2006; Ojala et al., 2000, [18] Zhao $\&$ etal, 2007 [11]) in a wide array of fields. Other fields where LBP has been recently investigated include face recognition (Ahonen et al., 2006 [3]; Nanni \& Lumini 2010 [12]; Zhao \& Pietikäinen, 2012 [8]; Zhang et al., 2007 [20]; Liao and Chung, 2007[9], facial expression detection (Shan et al., 2009) by Vijayakumar etal [5], remote-sensing image analysis (Lucieer et al., 2005) [14], and classification of biometrics (Nanni \& Lumini, 2008a) [17].

One of the main disadvantage of LBP is they are more sensitive to even a simple noise, this causes a change in LBP pattern. A LBP can generate 256 (0 to 255) different patterns. These patterns are grouped in to 59 uniform and 197 nonuniform LBP patterns. Many researchers have considered only uniform patterns for texture classifications due to their small numbers and claiming that most sof the textures are dominated by only uniform LBP's. An improvement suggested by Zhenhua Guo, Lei Zhang. [21] is to consider only the so-called

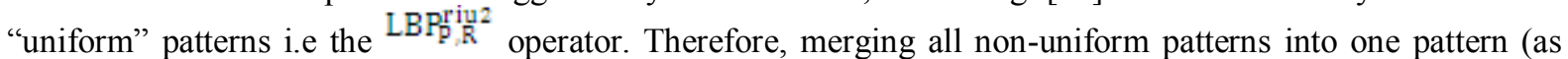
done in the traditional LBP) or some limited patterns (as done in Hui Zhou et al., 2008 [7]) do not describe the stochastic characteristics of texture efficiently. As a result, texture primitive information represented by these patterns is lost, especially when large neighborhoods are considered. This single pattern makes the uniform patterns sensitive to noise. A further improvement of this classical LBP operator $[2,13]$ is to make efficient use of non-uniform patterns in an appropriate way. To fulfill this purpose, all non-uniform patterns are classified into different subsets. It incorporates few nonuniform patterns with mostly uniform patterns in a feature vector. Combining few nonuniform patterns with a uniform patterns was shown in [2] to improve performance. Nonuniform patterns thus appear to contain useful information. However, the existing methods still suffer much from non-monotonic random noise, illumination variation.

Several attempts were made to use non-uniform patterns and overcome this limitation of the standard LBP (Heikkila et al., 2009 [6]; Zhou et al., 2008 [7]; Liao et al., 2009 [10]). Heikkila et al. (2009) [6] and Liao etal (2007) [9] extracted rotation invariant non-uniform patterns. Zhou et al. (2008) [7] proposed an extended version of LBP operator (LBP ${ }^{\text {extend }}$ ) which tried to use more than one bin for describing non-uniform patterns and to reduce the effect of noise. They used Hamming's distance to assign each LBP code (noisy code) to certain LBP codes (with minimum distance). Also Liao et al. (2009) [10] tried to extract the dominant (uniform and non-uniform) patterns (LBP ${ }^{\mathrm{dom}}$ ) which cover $80 \%$ of all the existing patterns in the input image as texture descriptor. They discarded the information on type of pattern to reduce the effect of noise. These extensions could solve this problem partially. 
But after a careful, critical and significant study on the existing literature, the present study found that ULBP have some shortcomings: they discard some important texture information, suffer much from nonmonotonic illumination variation and do not describe the stochastic characteristics of texture efficiently and sensitive to noise. To overcome this, the present thesis defined 'Semi Uniform LBP' (SULBP). To overcome the noise effect on LBP the present study derived 'Local Directional Pattern' (LDP) using Kirsch mask. The proposed study derived 'Semi Uniform patterns on LDP' (SULDP). The derived SULDP is an efficient image classification and analysis, which will have a significant role in image mining. The novelty of the proposed method is, the Haralick features are applied on the derived SULDP-CM, which has shown excellent classification results by reducing the overall dimension of the derived matrix dimension, thus reducing the overall complexity.

The paper is organized as follows. The basics of local binary pattern, uniform and Non-uniform patterns are discussed in section 2. In Section 3, contains proposed semi uniform LDP, experimental results and some discussions are in section 4 . Section 5 contains the concluding remarks.

\section{Local Binary Pattern and Uniform and Nonuniform Patterns}

Local Binary Pattern (LBP): Given a central pixel in the image, a pattern number is computed by comparing its value with its neighborhood values as shown in given Equation (1).

$$
L B P_{p, R}=\sum_{p=0}^{p-1} S\left(g_{p}-g_{c}\right) 2^{p}, \quad S(t)=\left\{\begin{array}{rr}
1 & t \geq 0 \\
0 & \text { otherwise }
\end{array}\right.
$$

where gray value $g_{c}$ corresponds to the gray value of the center pixel of the local neighborhood and $g_{p}$ $(p=0,1, \ldots, P-1)$ correspond to the gray values of $P$ equally spaced pixels on a circle of radius $R(R>0)$ that form a circularly symmetric neighbor set. Suppose the coordinates of $g_{c}$ are $(0,0)$, then the coordinates of $g_{p}$ are given by $(-R \sin (2 \Pi p / P), R \cos (2 \Pi p / P))$. The gray value of neighbour points which do not fall exactly in the centre of a pixel is calculated by linear interpolation of the corresponding pixels. Figurel shows an exemplary illustration for generation of LBP code.

\begin{tabular}{|l|l|l|}
\hline 85 & 32 & 26 \\
\hline 53 & 50 & 10 \\
\hline 60 & 38 & 48 \\
\hline
\end{tabular}

\begin{tabular}{|l|l|l|}
\hline 1 & 0 & 0 \\
\hline 1 & & 0 \\
\hline 1 & 0 & 0 \\
\hline
\end{tabular} LBP code $=00111000$

Figure 1: The basic LBP operator.

Uniform and Non Uniform LBP: An LBP generates a total of 256 patterns ranging from 0 to 255. Moreover, it is easy to realize that due to the way LBP numbers are created, they are very sensitive to noise: the slightest fluctuation above or below the value of the central pixel is treated the same way as a major contrast between the central pixel and its surroundings. Considering such a huge size of patterns for classification purpose is a very complex task and tedious process. To overcome this LBP patterns are divided in to uniform and non uniform patterns.

An important special case of LBP is the 'Uniform LBP' (ULBP). A pattern is considered 'uniform' (U) if the number of bit transactions between 0 and 1 is less than or equal to two. It was observed by Ojala et al. [15, 16] that certain patterns seem to be fundamental properties of texture, these patterns are called "uniform" because they have at most two one-to-zero or zero-to-one transitions in the circular binary code defined in Equation (2). That is, the larger the uniformity value $U$ is, the more likely is that spatial transition occurs in the local pattern.

$$
\begin{aligned}
& U\left(L B P_{p R}\right)=\left|s\left(q_{p-1}-q_{c}\right)-s\left(q_{0}-q_{c}\right)\right|+ \\
& \sum p=1\left|s\left(q_{p}-q_{c}\right)-s\left(q_{p-1}-q_{c}\right)\right|
\end{aligned}
$$

For example, patterns 0 (bitwise 00000000) and 255 (bitwise 11111111) have a U value of 0 while patterns $1,2,4,8,16,32,64$ and 128 (bitwise 00000001, 00000010, etc.) have a $U$ value of 2 as there are exactly two $0 / 1$ or $1 / 0$ transitions in the bitwise representation. Similarly, patterns 00000011, 00000111, 00001111, 00011111, 00111111, 01111111 and other circularly rotated bitwise rotated versions have a U value of 2. Finally, patterns of (00000000, 00000001, 00000011, 00000111, 00001111, 00011111, 00111111, 0111111111111111 ) and their circularly rotated versions of transformation invariant analysis have a $U$ value of 2. These nine patterns correspond to $\mathrm{P} \times(\mathrm{P}-1)+3$ distinct output values (i.e., 58 of $3 \times 3$ neighbourhood) of the $2^{\mathrm{P}}$ un-rotated patterns that can occur in the $3 \times 3$ neighbourhood. An important observation was made by Ojala et al. [15] that in texture images, majority of LBP features can be categorized to be uniform. In practice, all the nonuniform patterns $(\mathrm{U}>2)$ are grouped under a "miscellaneous" label, while each uniform LBP is cast into a 
unique histogram bin according to its decimal value. That is the total number of non uniform patterns are $256-$ $59=197$. Non uniform patterns fall in to a large category of patterns. It was verified that only "uniform" patterns are fundamental patterns of local image texture.

However, all the above patterns still have some shortcomings: they discard some important texture information, suffer much from non-monotonic illumination variation, do not describe the stochastic characteristics of texture efficiently and sensitive to noise. As a result of this texture primitive information represented by these patterns is lost.

In order to overcome these shortcomings, the present paper defined semi uniform LBP. While, the LBP pattern, 01010000 (4 transitions), 01110101 (6 transitions) are non-uniform ones. However, the existing LBP operators can't use the information of non-uniform patterns efficiently because they consist of more number of patterns. To overcome this, the present paper thought that prospective improvement is required for the efficient use of non-uniform patterns in an appropriate way.

\section{Proposed Semi-Uniform Lbp (Sulbp)}

The present study defined Semi-uniform LBP as those patterns of LBP with exactly four bitwise transitions $(\mathrm{U}=4)$. Further the proposed study derived the semi-uniform patterns are those patterns of LBP that will have at least two one's (such as 00100010 for $\mathrm{P}=8$ ) and at most have $\mathrm{P}-2$ one's (such as 11011101 for $\mathrm{P}=8$ ).

Local Directional Pattern: The present paper evaluates Semi uniform patterns on LDP instead of LBP for the following reasons.

- LDP overcome sensitive to random noise and non-monotonic to illumination variation problems in LBP since edge response magnitude is more stable than pixel intensity.

- LDP descriptor effectively uses the texture information contained in the Semi- LBP patterns, and then becomes more robust against noise.

The present paper derived LDP technique with 'Kirsch Edge Response' (KER). The LDP is an eight bit binary code assigned to each pixel of an input image. The local descriptor LDP considers the edge response values in all different directions instead of surrounding neighboring pixel intensities like LBP. This provides more consistency in the presence of noise, and illumination changes, since edge response magnitude is more stable than pixel intensity. The LDP is based on LBP. The Kirsch masks in eight different orientations $\left(\mathrm{M}_{0} \sim \mathrm{M}_{7}\right)$ centered on its own position are given in Figure 2.

$$
\begin{aligned}
& {\left[\begin{array}{ccc}
-3 & -3 & 5 \\
-3 & 0 & 5 \\
-3 & -3 & 5
\end{array}\right]\left[\begin{array}{ccc}
-3 & 5 & 5 \\
-3 & 0 & 5 \\
-3 & -3 & -3
\end{array}\right]\left[\begin{array}{ccc}
5 & 5 & 5 \\
-3 & 0 & -3 \\
-3 & -3 & -3
\end{array}\right]\left[\begin{array}{ccc}
5 & 5 & -3 \\
5 & 0 & -3 \\
-3 & -3 & -3
\end{array}\right]}
\end{aligned}
$$

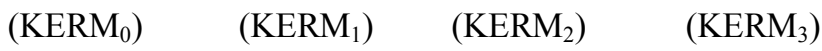

$$
\begin{aligned}
& {\left[\begin{array}{ccc}
5 & -3 & -3 \\
5 & 0 & -3 \\
5 & -3 & -3
\end{array}\right]\left[\begin{array}{ccc}
-3 & -3 & -3 \\
5 & 0 & -3 \\
5 & 5 & -3
\end{array}\right]\left[\begin{array}{ccc}
-3 & -3 & -3 \\
-3 & 0 & -3 \\
5 & 5 & 5
\end{array}\right]\left[\begin{array}{ccc}
-3 & -3 & -3 \\
-3 & 0 & 5 \\
-3 & 5 & 5
\end{array}\right]} \\
& \left(\mathrm{KERM}_{4}\right) \quad\left(\mathrm{KERM}_{5}\right) \quad\left(\mathrm{KERM}_{6}\right) \quad\left(\mathrm{KERM}_{7}\right)
\end{aligned}
$$

Figure 2: Kirsch edge response masks (KERM) in eight directions.

The LDP is derived by applying the above Kirsch Edge Response Mask (KERM) from $\mathrm{KERM}_{0}$ to $\mathrm{KERM}_{7}$, on each $3 \times 3$ non overlapping window. The value obtained by applying the $\mathrm{KERM}_{0}$ on a $3 \times 3$ mask determines the grey level pixel value at position $\mathrm{g}_{0}$. This grey level value is named as $\mathrm{KER}_{0}$ value at position $\mathrm{g}_{0}$. In the same way by applying the $\mathrm{KERM}_{7}$ on the same $3 \times 3$ mask determine the grey level pixel value at position $\mathrm{g}_{7}$. That is by applying eight $\mathrm{KERM}$ from 0 to 7 , eight $\mathrm{KER}_{0: 7}$ values are obtained and positioned at $\mathrm{g}_{0: 7}$ pixel locations respectively as shown in Figure 3.

\begin{tabular}{|l|l|l|}
\hline $\mathrm{g}_{3}$ & $\mathrm{~g}_{2}$ & $\mathrm{~g}_{1}$ \\
\hline $\mathrm{g}_{4}$ & & $\mathrm{~g}_{0}$ \\
\hline $\mathrm{g}_{5}$ & $\mathrm{~g}_{6}$ & $\mathrm{~g}_{7}$ \\
\hline
\end{tabular}

$=$\begin{tabular}{|l|l|l|}
\hline $\mathrm{KER}_{3}$ & $\mathrm{KER}_{2}$ & $\mathrm{KER}_{1}$ \\
\hline $\mathrm{KER}_{4}$ & & $\mathrm{KER}_{0}$ \\
\hline $\mathrm{KER}_{5}$ & $\mathrm{KER}_{6}$ & $\mathrm{KER}_{7}$ \\
\hline
\end{tabular}

Figure 3. Transformation matrix from gray level image into KER matrix 
Assume a $3 \times 3$ original matrix as shown in Figure 4 . The transformation process by applying the above KERM's on the original matrix is shown below. Figure 5 shows step by step mechanism of this transformation process

\begin{tabular}{|c|c|c|}
\hline 85 & 32 & 26 \\
\hline 53 & 50 & 10 \\
\hline 60 & 38 & 48 \\
\hline
\end{tabular}

Figure 3: Original $3 \times 3$ matrix

\begin{tabular}{|l|l|l|}
\hline 85 & 32 & 26 \\
\hline 53 & 50 & 10 \\
\hline 60 & 38 & 48 \\
\hline
\end{tabular}

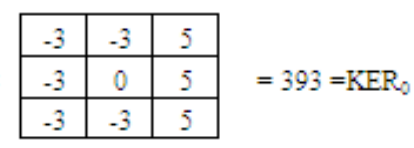

\begin{tabular}{|c|c|c|}
\hline 85 & 32 & 26 \\
\hline 53 & 50 & 10 \\
\hline 60 & 38 & 48 \\
\hline
\end{tabular}$\times$\begin{tabular}{|c|c|c|}
\hline-3 & 5 & 5 \\
\hline-3 & 0 & 5 \\
\hline-3 & -3 & -3 \\
\hline
\end{tabular}$=503=\mathrm{KER}_{1}$

\begin{tabular}{|c|c|c|}
\hline 85 & 32 & 26 \\
\hline 53 & 50 & 10 \\
\hline 60 & 38 & 48 \\
\hline
\end{tabular}$\times$\begin{tabular}{|c|c|c|}
\hline 5 & 5 & 5 \\
\hline-3 & 0 & -3 \\
\hline-3 & -3 & -3 \\
\hline
\end{tabular}$\quad=97=\mathrm{KER}_{2}$
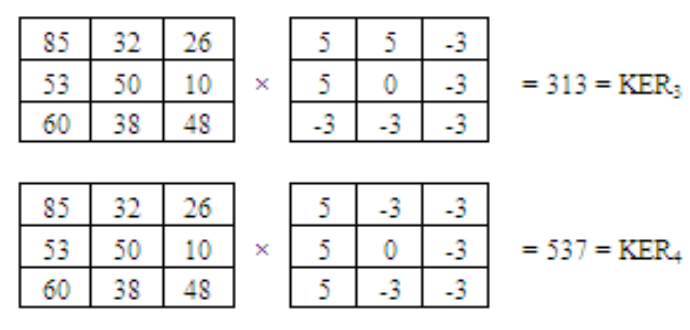

\begin{tabular}{|c|c|c|}
\hline 85 & 32 & 26 \\
\hline 53 & 50 & 10 \\
\hline 60 & 38 & 48 \\
\hline 5 & 5 & -3 \\
\hline 5 & 0 & -3 \\
\hline-3 & -3 & -3 \\
\hline
\end{tabular}$=161=\mathrm{KER}_{3}$

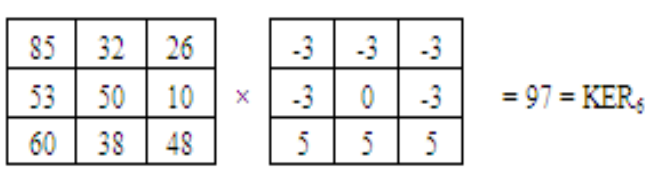

\begin{tabular}{|c|c|c|}
\hline 85 & 32 & 26 \\
\hline 53 & 50 & 10 \\
\hline 60 & 38 & 48 \\
\hline
\end{tabular}$\times$\begin{tabular}{|c|c|c|}
\hline-3 & -3 & -3 \\
\hline-3 & 0 & 5 \\
\hline-3 & 5 & 5 \\
\hline
\end{tabular}$=161=\mathrm{KER}$ ?

Figure 5: Step by step process of grey level values obtained by applying KERM on the $3 \times 3$ matrix of Figure 4 .

Once the step by step process is completed, the Figure 6 gives the KER matrix. The Figure 6 is formed based on the step wise procedure of Figure 5. From Figure 6(c), the LDP code is obtained based on the Equation (3), which is given below. The LDP code is 11011000

$$
L D R_{k}=\sum_{i=0}^{7} b\left(m_{i}-m_{k}\right) 2^{i}, \quad b_{i}(t)=\left\{\begin{array}{lr}
1 & t \geq 0 \\
0 & \text { otherwise }
\end{array}\right.
$$

Where, $\mathrm{M}_{\mathrm{k}}$ is the $\mathrm{k}^{\text {th }}$ most significant directional response in order to generate the LDP. The values of $\left|\mathrm{m}_{\mathrm{j}}\right|$ are nothing but the top $\mathrm{k}$ values set them to 1 , remaining $(8-\mathrm{k})$ bits are set to 0 


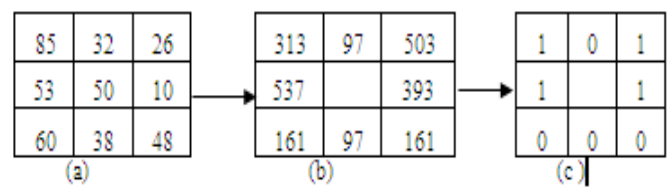

Figure 6: a) Original gray level matrix b) The proposed KER matrix $\quad$ c) The LDP.

The advantage of LDP over LBP is explained with the following example. In the Figure 7 a small noise changes the LBP value drastically. But this noise effect is not sensed by LDP because of its KER gray level values. After addition of noise, the $5^{\text {th }}$ bit of LBP is changed from 1 to 0 , thus LBP pattern changed from uniform to a non-uniform code. A small one bit error or a small noise may change uniform LBP into nonuniform LBP or vice versa. Since gradients are more stable than gray value, LDP pattern provides the same pattern value even in the presence of noise and non-monotonic illumination changes.

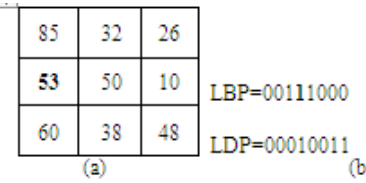

Figure 7: Stability of LDP vs. LBP (a) Original image (b) Image with noise

A histogram is generated to represent, analyze, and characterize texture images using LDP. So, after computing all the LDP code for every pixel $(\mathrm{r}, \mathrm{c})$, the input image $\mathrm{L}$ of size $\mathrm{M} \times \mathrm{N}$ is represented by a LDP histogram $\mathrm{H}$ using Equation (4). The resultant histogram $\mathrm{H}$ is the LDP descriptor of that image.

$$
\begin{aligned}
& H(k)=\sum_{r=1}^{M} \sum_{\mathrm{r}=1}^{\mathrm{N}} \mathrm{f}\left(\mathrm{L}_{k}(\mathrm{r}, \mathrm{c}), \mathrm{y}\right), \quad \mathrm{f}(\mathrm{x}, \mathrm{y})= \\
& \left\{\begin{array}{l}
1 \\
0 \text { otherwise }
\end{array}\right.
\end{aligned}
$$

where $\mathrm{y}$ is the LDP code value. This histogram forms the LDP feature vector, which characterizes image texture. A histogram is then constructed to collect the occurrences of uniform patterns and hence the $\mathrm{LDP}_{3 \times 3}$ histogram has 59 bins

\section{Experimental Results}

To evaluate the effectiveness of the proposed SULDP method, experiments are conducted on two different datasets. The first dataset i.e., Dataset- 1 is composed of 45 texture classes (one image for each class) from the OuTex library [22]. The second dataset, i.e., Dataset-2 is composed of 12 granite texture classes [4]. The overall dataset is composed of 48 images, 4 for each class.

The performance of the proposed SULDP is tested in the two datasets for classification of textures. For the classification, leave one out classifier is used to compare the correct classification percentage of the proposed SULDP method along with LBP ${ }^{\mathrm{dom}}$ and LBP ${ }^{\text {extend }}$ in three different situations as below:

Setup 1: The training set considers only the original sub images (without rotation) and the others are used for testing. (Approximately $12 \%$ were used for training and $88 \%$ for testing)

Setup 2: The training set considers of sub images with rotation angle of $0^{\circ}, 45^{\circ}, 90^{\circ}, 135^{\circ}$ and, $180^{\circ}$ (approximately $25 \%$ were used for training and $75 \%$ for testing).

Setup 3: The training set considers of half of all sub images that are selected randomly (50\% were used for training and $50 \%$ for testing).

These experiments are repeated for each method with $(\mathrm{P}=8, \mathrm{R}=1)$ and $(\mathrm{P}=16, \mathrm{R}=2)$, and also with a combination of them. Table 1 shows the obtained results of these experiments.

Table 1: mean (\%) classification results of the proposed SULDP method of Dataset-1 and Dataset-2.

\begin{tabular}{|c|c|c|c|c|c|c|}
\hline \multirow{2}{*}{ Method Type } & \multicolumn{3}{|c|}{ OuTex Database } & \multicolumn{3}{c|}{ Granite Database } \\
\cline { 2 - 7 } & Setup 1 & Setup 2 & Setup 3 & Setup 1 & Setup 2 & Setup 3 \\
\hline SULDP & 45.4 & 70.5 & 90.7 & 46.5 & 76.9 & 93.5 \\
\hline SULDP $_{16,2}$ & 53.6 & 80.1 & 93.5 & 49.4 & 87.3 & 95.1 \\
\hline SULDP $_{8,1} \&$ SULDP $_{16,2}$ & 48.2 & 91.4 & 95.9 & 50.8 & 84.6 & 96.5 \\
\hline
\end{tabular}

From the obtained results, the experiment Setup 1 did not result in good classification accuracies for the two datasets; all methods have accuracy less than $75 \%$, because the number of samples for training the $\mathrm{L}_{1}$ norm classifier is too small. The experiment Setup 2 gave better classification accuracies than the experiment Setup 1, because it used more samples for training. However, there are many differences between the obtained 
classification performances of Setup 1 and Setup 2. Regardless of which experimental setup was used, the proposed method gave the best performance on the both datasets, and also better classification accuracy can be obtained only by combining the operators with enough training samples (it was greater than $95 \%$ for the OuTex dataset and Granite dataset in experiment Setup 3).

\section{Comparison of the Proposed SULDP Method with other Existing Methods}

The proposed SULDP is compared with ${ }^{{ }^{L B} P_{8,1}^{\text {dom }}}[10],{ }^{{ }^{L B P} P_{8,1}^{e x t e n d ~}}$ [7] methods. Mean classification rates for the proposed SULDP and the other existing methods using K-NN classifier is shown in Table 2 which clearly indicates that the proposed SULDP outperforms the other existing methods with two different scales(S).

Table 2: Comparison of the proposed SULDP method with the other existing methods.

\begin{tabular}{|c|c|c|c|c|c|c|}
\hline \multirow{2}{*}{ Method Type } & \multicolumn{3}{|c|}{ OuTex Database } & \multicolumn{3}{|c|}{ Granite Database } \\
\hline & Setup! & Setup2 & Setup3 & Setup! & Setup2 & Setup? \\
\hline $\operatorname{LBP}_{3,1}^{\mathrm{dom}}[10]$ & 43.2 & 65.8 & 67.5 & 39.6 & 61.7 & 69.4 \\
\hline $\operatorname{LBP}_{8,1}^{\text {extend }}[7]$ & 35.3 & 69.7 & 71.4 & 41.8 & 55.6 & 67.3 \\
\hline $\operatorname{SULDP}_{8,1}$ & 45.4 & 70.5 & 90.7 & 46.5 & 76.9 & 93.5 \\
\hline LBP dom & 48.2 & 67.5 & 73.6 & 49.3 & 73.9 & 72.4 \\
\hline $\operatorname{LBP}_{16,2}^{\text {extand }}[7]$ & 38.1 & 68.3 & 91.6 & 45.4 & 73.4 & 86.7 \\
\hline SULDPP $_{16,2}$ & 53.6 & 80.1 & 93.5 & 49.4 & 87.3 & 95.1 \\
\hline $\mathrm{LBP}_{3,1}^{\mathrm{dom}} \& \mathrm{LBP} P_{16,2}^{\mathrm{dom}}[10]$ & 49.8 & 84.8 & 91.7 & 57.9 & 78.8 & 80.6 \\
\hline $\mathrm{LBP}_{8,1}^{\text {extand }} \& \mathrm{LBP}_{16,2}^{\text {etrand }} \quad[7]$ & 49.2 & 88.5 & 92.8 & 47.6 & 82.4 & 87.9 \\
\hline $\operatorname{SULDP}_{8,1} \& \operatorname{SULDP}_{16,2}$ & 48.2 & 91.4 & 95.9 & 50.8 & 84.6 & 96.5 \\
\hline
\end{tabular}

\section{Conclusion}

The LBP operator is a powerful texture descriptor for image analysis. This operator can extract statistical and structural texture features. The traditional $\mathrm{LBP}_{\mathrm{P}, \mathrm{R}}^{\mathrm{M}} \mathrm{uses}$ only uniforms patterns and merge all nonuniform patterns into one class. The traditional LBP has two weak points: first, it cannot describe texture characteristics efficiently, because it concentrates only on the uniform patterns which are representing the regular components of texture. It merges all non-uniform patterns into one label and discards stochastic information of texture patterns. Therefore, the performance of the traditional LBP operator deteriorates, especially in analysis of the texture of natural images. Second is that it is sensitive to noise. When noise varies at least one pixel the label of patterns are changed. One way to avoid noisy patterns, the present study proposed a new concept called 'Local Directional Pattern' (LDP) descriptor. LDP descriptor is more consistent in the presence of noise and illumination changes, since edge response magnitude is more stable than pixel intensity.

\section{References}

[1] T., Pietikäinen M., Mäenpää T., "Multi-resolution gray-scale and rotation invariant texture classification with local binary patterns", IEEE Trans. Pattern Anal., vol.24, pp.971-987, 2002.

[2] Abdolhossein Fathi, Ahmad Reza Naghsh-Nilchi, "Noise tolerant local binary pattern operator for efficient texture analysis", Pattern Recognition Letters, vol.33, pp.1093-1100, 2012.

[3] Ahonen T., Hadid A., Pietikäinen M., "Face description with local binary patterns: application to face recognition", IEEE Transactions on Pattern Analysis and Machine Intelligence, vol.28(12), pp.2037-2041, 2006.

[4] Antonio Fern'andez, Ovidiu Ghita, Elena Gonz'alez, Francesco Bianconi, Paul F. Whelan, "Evaluation of robustness against rotation of LBP, CCR and ILBP features in granite texture classification”, Machine Vision and Applications, 2011.

[5] Eswara Reddy B., Chandra Sekhar Reddy P., Vijaya Kumar V., "Texton based shape features on local binary pattern for age classification”, Int. Jour. graphics and signal processing, pp.110-116, 2012.

[6] Heikkila, M., Pietikäinen, M., Schmid, C., "Description of interest regions with local binary patterns", Pattern Recognition, vol.42, pp.425-436, 2009.

[7] Hui Zhou, Runsheng Wang, Cheng Wang, "A novel extended local-binary-pattern operator for texture analysis", Information Sciences, vol.178 pp.4314-4325, 2008 .

[8] Li Liu, Lingjun Zhao, Yunli Long, Gangyao Kuang, Paul Fieguth, "Extended local binary patterns for texture classification”, Image and Vision Computing, pp.1-14, 2012.

[9] Liao S., Chung A.C.S., "Face recognition by using Elongated local binary patterns with average maximum distance gradient magnitude", Computer Vision- ACCV, pp.672-679, 2007

[10] Liao S., Law M.W.K., Chung A.C.S., "Dominant local binary patterns for texture classification", IEEE Transactions on Image Processing, vol.18(5), pp.1107-1118, 2009. 
[11] Liao S., Zhu X., Lei Z., Zhang L., Li S., "Learning multi-scale block local binary patterns for face recognition", in: International Conference on Biometrics, ICB07, pp.828-837, 2007.

[12] Loris Nanni, Sheryl Brahnam, Alessandra Lumini, "A local approach based on a Local Binary Patterns variant texture descriptor for classifying pain states”, Expert Systems with Applications, vol.37, pp.7888-7894, 2010.

[13] Loris Nanni, Sheryl Brahnam, Alessandra Lumini, “A simple method for improving local binary patterns by considering nonuniform patterns", Pattern Recognition, pp.1-9, 2012.

[14] Lucieer A., Stein P., Fisher, "Multivariate texture-based segmentation of remotely sensed imagery for extraction of objects and their uncertainty", International Journal of Remote Sensing, vol.26(14), pp.2917-2936, 2005.

[15] MaÈenpaĖaĖ T., Ojala T., PietikaĖinen M., and Soriano M., "Robust texture classification by subsets of local binary patterns", Proc. 15th Int'l Conf. Pattern Recognition, vol.3, pp. 947-950, 2000.

[16] MaÈenpaÈaÈ T., PietikaĖinen M., and Ojala T., "Texture Classification by Multi-Predicate Local Binary Pattern Operators", Proc. $15^{\text {th }}$ Int'l Conf. Pattern Recognition, vol.3, pp.951-954, 2000.

[17] Nanni L., Lumini A., "Local binary patterns for a hybrid fingerprint matcher”, Pattern Recognition, vol.41, pp.3461-3466, 2008.

[18] Ojala T., Pietikäinen M., Mäenpää T., "Gray scale and rotation invariant texture classification with local binary patterns", Proceedings of Sixth European conference on Computer Vision, pp.404-420, 2000.

[19] Ahonen T., Hadid A., Pietikäinen M., "Face description with local binary patterns: Application to Face Recognition," IEEE Trans. PAMI, vol.28(12), pp:2037-2041, 2006.

[20] Zhang W., Shan H., Chen X., Gao W., "Local gabor binary patterns based on mutual information for face recognition", Int.Journal of Image Graphics, vol.7(4), pp.777-93, 2007.

[21] Zhenhua Guo, Lei Zhang, David Zhang, "Rotation invariant texture classification using LBP variance (LBPV) with global matching", Pattern Recognition, vol.43, pp.706-719, 2010.

[22] Ojala T., Pietikäinen M., Mäenpää T., Viertola J., Kyllönen J., Huovinen S., "Outex-new framework for empirical evaluation of texture analysis algorithms,” In: Proc. 16th Int. Conf. on Pattern Recognition, vol.1, pp:701-706, 2002(b). 